\title{
DNA mismatch repair proteins: scientific update and practical guide
}

\author{
Adrian C Bateman
}

Correspondence to

Dr Adrian C Bateman, Cellular

Pathology, University Hospital Southampton NHS Foundation Trust, Southampton S016 6YD UK; adrian.bateman@uhs.

nhs.uk

Received 24 November 2020 Revised 23 January 2021 Accepted 25 January 2021 Published Online First 17 February 2021
Check for updates

(c) Author(s) (or their employer(s)) 2021. No commercial re-use. See rights and permissions. Published by BMJ.

To cite: Bateman AC. J Clin Pathol

$2021 ; 74: 264-268$

\section{ABSTRACT}

DNA mismatch repair (MMR) proteins are essential for the recognition and correction of sporadic genetic mutations that occur during DNA replication. Deficient MMR function (dMMR) leads to an increased risk of development of neoplasia. Identification of dMMR within tumours can suggest a high chance of the inherited cancer condition Lynch syndrome and predicts poor clinical response to certain conventional chemotherapies but an increased likelihood of response to immunotherapy. This review provides an update on the biology of MMR proteins, their encoding genes and mechanisms for the development of dMMR. This is followed by a discussion of the identification and significance of dMMR in routine clinical practice.

\section{MISMATCH REPAIR PROTEINS AND THEIR FUNCTION}

Mismatch repair (MMR) proteins are essential for repairing DNA errors (eg, point mutations) that are generated during DNA replication. There are at least seven MMR proteins in humans, of which four have the most clinical relevance in human cancer biology-MLH-1, MSH-2, MSH-6 and PMS-2. ${ }^{1}$ These four proteins are arranged as heterodimers in which MLH-1 associates with PMS-2 and MSH-2 associates with MSH-6. The MLH-1/PMS-2 pairing recognises mismatched nucleotide base pairs and initiates repair, while the MSH-2/MSH-6 pairing acts as an endonuclease. ${ }^{2}$ These proteins are encoded by their corresponding genes (MMR genes)-MLH-1, MSH-2, MSH-6 and PMS-2.

\section{CONSEQUENCES OF LOSS OF MMR PROTEIN FUNCTION}

Loss of function of one or more MMR proteins (deficient MMR (dMMR)) leads to impaired DNA repair capability. This results in the accumulation of spontaneous genetic mutations across the genome, affecting the function of many genes. Those involved in normal cellular growth and differentiation (proto-oncogenes) may become amplified (present at multiple copy numbers) or undergo gain-of-function mutations (creating oncogenes), while the function of tumour suppressor genes may be impaired. This leads to an increased risk of development of neoplasia. Identification of dMMR has two main areas of clinical relevance-screening for inherited cancer syndromes, for example, Lynch syndrome and the prediction of response to conventional chemotherapy and immunotherapy.

Lynch syndrome was first described by the American physician Henry T Lynch in 1966 and is a familial cancer condition that is almost always associated with the presence of a mono-allelic germline mutation in an MMR gene. ${ }^{3}$ Affected patients are at increased risk of development of one or more of a range of neoplasms (table 1). The syndrome was originally termed 'hereditary non-polyposis colorectal cancer (CRC)' due to its common association with CRC. However, recognition of the broad range of possible tumour types has resulted in its renaming as 'Lynch syndrome'. Around 50\% of patients with Lynch syndrome have neoplasms other than CRC or endometrial carcinoma. ${ }^{4}$ Certain other tumours may rarely be associated with Lynch syndrome but the evidence supporting their inclusion within the spectrum of Lynch-associated neoplasms is currently weak, for example, breast cancer. ${ }^{5}$

\section{MECHANISMS OF DMMR DEVELOPMENT}

dMMR function can occur due to a mutation within an $M M R$ gene or because of inactivation of an otherwise intact $M M R$ gene. $M M R$ gene mutations may be present in germline DNA or can occur as somatic events within a tumour. Mono-allelic germline $M M R$ gene mutations are associated with Lynch syndrome but bi-allelic germline mutations may very rarely be encountered and lead to 'constitutional MMR deficiency' or 'childhood cancer syndrome. ${ }^{6}$

Inactivation of $M M R$ genes occurs with $M L H-1$ and is a consequence of hypermethylation of the promoter sequence of the gene. Hypermethylation is a common biological process that comprises the addition of methyl groups $\left(-\mathrm{CH}_{3}\right)$ to $\mathrm{CpG}$ islands. The latter are DNA regions where a cytosine nucleotide is followed by a guanine nucleotide at a high frequency-the ' $\mathrm{p}$ ' indicates that the two nucleotides are separated by only a phosphate group that is, that they are present on a single strand of DNA. Hypermethylation is a physiological process for controlling gene expression and many sites within the genome are methylated during cellular development and differentiation. However, most $\mathrm{CpG}$ islands within promoter sequence DNA are usually unmethylated. Hypermethylation of promoter sequences causes inactivation of the promoter and switches off the corresponding gene. Tumours such as CRC and precursor lesions showing these genetic changes are described as 'CIMP-high' (ie, $\mathrm{CpG}$ island methylation phenotype-high level).

Hypermethylation is usually a somatic process and within colorectal neoplasia, occurs commonly in the serrated pathway of carcinogenesis in association with BRAF mutations. Constitutional 


\begin{tabular}{|c|c|}
\hline Cancer type & Notes \\
\hline \multicolumn{2}{|l|}{ Gastrointestinal } \\
\hline Colorectal carcinoma $(\mathrm{CRC})^{*}$ & Accounts for $3 \%-5 \%$ of all CRC \\
\hline \multicolumn{2}{|l|}{ Gastric adenocarcinoma } \\
\hline \multicolumn{2}{|l|}{ Small intestinal adenocarcinoma } \\
\hline \multicolumn{2}{|l|}{ Pancreatic adenocarcinoma } \\
\hline \multicolumn{2}{|l|}{ Cholangiocarcinoma } \\
\hline \multicolumn{2}{|l|}{ Gynaecological } \\
\hline Endometrial carcinoma* & $\begin{array}{l}\text { Accounts for } 2 \%-3 \% \text { of all endometrial } \\
\text { cancers }\end{array}$ \\
\hline \multicolumn{2}{|l|}{ Ovarian carcinoma } \\
\hline \multicolumn{2}{|l|}{ Other sites } \\
\hline \multicolumn{2}{|c|}{ Urinary tract carcinoma (transitional cell) } \\
\hline \multicolumn{2}{|l|}{ Prostatic carcinoma } \\
\hline Cutaneous sebaceous tumours $\dagger$ & Muir-Torre syndrome \\
\hline \multicolumn{2}{|l|}{ Glioblastoma } \\
\hline \multicolumn{2}{|l|}{ Adrenocortical carcinoma } \\
\hline \multicolumn{2}{|l|}{ Germ cell tumours } \\
\hline \multicolumn{2}{|l|}{ Mesothelioma } \\
\hline \multicolumn{2}{|l|}{ Melanoma } \\
\hline Sarcoma & \\
\hline
\end{tabular}

* CRC and endometrial carcinoma are the two neoplasms most commonly associated with Lynch syndrome.

tThere is also an increased risk of development of keratoacanthoma.

hypermethylation can rarely occur and may be heritable in a Mendelian fashion, depending on the precise nature of $M M R$ gene inactivation present. $^{7}$

\section{IDENTIFICATION OF DMMR IN DIAGNOSTIC PRACTICE}

In clinical practice, $\mathrm{dMMR}$ may be detected at a genetic, protein or functional level. Diagnostic histopathologists will be most familiar with the use of immunohistochemistry (IHC) to demonstrate the presence of MMR proteins within tumour cell nuclei. Due to MMR protein heterodimerism, the pattern of loss of expression of the four most commonly studied proteins provides useful clues to the most likely underlying genetic defect (table 2).

\begin{tabular}{|c|c|}
\hline Pattern of expression loss on IHC & Likely clinical implication \\
\hline \multicolumn{2}{|l|}{ Common patterns } \\
\hline MLH-1 and PMS-2 & $\begin{array}{l}\text { MLH-1 inactivation } \\
M L H-1 \text { mutation }\end{array}$ \\
\hline MSH2 and MSH-6 & $\begin{array}{l}\text { MSH-2 mutation } \\
\text { EPCAM mutation (less common) }\end{array}$ \\
\hline MSH-6 alone* & $\begin{array}{l}\text { MSH- } 6 \text { mutation } \\
\text { MSH-2 mutation (less common) }\end{array}$ \\
\hline PMS-2 alonet & $\begin{array}{l}\text { PMS-2 mutation } \\
\text { MLH-1 mutation (less common) }\end{array}$ \\
\hline \multicolumn{2}{|l|}{ Rare patterns } \\
\hline MLH-1 alone & $\begin{array}{l}\text { MLH-1 inactivation } \\
\text { MLH-1 mutation }\end{array}$ \\
\hline MSH-2 alone & $\begin{array}{l}\text { MSH-2 mutation } \\
\text { EPCAM mutation }\end{array}$ \\
\hline
\end{tabular}

${ }^{*} \mathrm{MSH}-2$ can form heterodimers with MMR proteins other than MSH-6 and therefore MSH-2 expression is usually not lost when an MSH-6 mutation is present.

TMLH-1 can form heterodimers with MMR proteins other than PMS-2 and therefore MLH-1 expression is usually not lost when a PMS-2 mutation is present. IHC, immunohistochemistry; MMR, mismatch repair.
Table 3 Commonly encountered difficulties in MMR protein immunohistochemistry interpretation

\begin{tabular}{ll}
\hline Interpretation difficulty & Comment \\
\hline $\begin{array}{l}\text { Weak MMR protein staining } \\
\text { throughout the tumour and in } \\
\text { stromal and inflammatory cells } \\
\text { (internal controls) }\end{array}$ & $\begin{array}{l}\text { Compare staining in tumour cells to that } \\
\text { within internal controls. If both compartments } \\
\text { are weak this is likely to be technical for } \\
\text { example, poor antigen preservation. Be } \\
\text { cautious before diagnosing dMMR in this } \\
\text { situation }\end{array}$ \\
$\begin{array}{ll}\text { Absent MMR protein staining in the } \\
\text { tumour and internal controls }\end{array}$ & $\begin{array}{l}\text { This is most likely to be technical failure } \\
\text { but in the appropriate clinical context also } \\
\text { consider the possibility of 'constitutional MMR } \\
\text { deficiency' }\end{array}$ \\
\hline $\begin{array}{l}\text { Patchy loss of MMR protein staining } \\
\text { in the tumour }\end{array}$ & $\begin{array}{l}\text { Compare staining in tumour cells to that } \\
\text { within internal controls. If both compartments } \\
\text { are weak this is likely to be technical but if the } \\
\text { internal controls are well stained in the same } \\
\text { area then this is likely to be due to a clonal } \\
\text { genetic event affecting the corresponding } \\
M M R \text { gene }\end{array}$ \\
\hline $\begin{array}{l}\text { Cytoplasmic MMR protein expression } \\
\text { within tumour }\end{array}$ & $\begin{array}{l}\text { This can occur when an } M M R \text { gene mutation } \\
\text { is present and the abnormal protein } \\
\text { accumulates within the cytoplasm }\end{array}$ \\
\hline
\end{tabular}

dMMR, deficient MMR; MMR, mismatch repair.

It has been suggested that an IHC panel limited to MSH-6 and PMS-2 may be a more cost-effective way of detecting dMMR. ${ }^{8-10}$ As well as identifying patients with a somatic or germline $\mathrm{MSH}-6$ or PMS-2 mutation, loss of PMS-2 or MSH-6 expression also occurs in those with a somatic or germline $\mathrm{MLH}-1$ or $\mathrm{MSH}-2$ mutation (or $\mathrm{MLH}-1$ inactivation), respectively. Isolated loss of MLH-1 or MSH-2 expression is rare. If this two-antibody panel is used, IHC for the 'paired' MMR protein could be performed if loss of MSH-6 or PMS-2 is found, in order to determine which $M M R$ gene is most likely to be defective. In practice, many laboratories still include all four of these MMR proteins in their IHC panel as the overall cost is still relatively low and the ability to assess both MMR proteins within each 'pair' can be useful when the staining quality is suboptimal, for example, due to poor initial tissue fixation.

Some of the more commonly encountered difficulties in the interpretation of MMR protein expression are described in table 3. In Lynch (and 'Lynch-like') syndrome, loss of MMR protein expression usually occurs in a widespread and homogeneous manner within the tumour. However, heterogeneity of MMR protein expression may be encountered and manifests as a defined area of lost expression in the presence of retained stromal and inflammatory cell MMR protein staining-within a tumour that otherwise shows widespread expression of the protein(s) concerned. In this situation, an individual malignant gland can sometimes show zones of retained and lost MMR protein expression. Assuming that technical reasons for variations in staining intensity have been excluded, this heterogeneous staining implies the presence of a clonal somatic $M M R$ gene mutation or localised hypermethylation process, that is, a mutation or genetic inactivation affecting only a small area of the tumour. Importantly, it does not imply that a germline mutation is present. ${ }^{11}$

Microsatellites are non-coding DNA regions that are present throughout the genome and that-along with coding regionsare replicated imperfectly during cell division if there is dMMR. These imperfections can be identified within tumour-derived DNA using PCR-based testing and this phenomenon is termed 'microsatellite instability' (MSI). MSI therefore represents an 
alternative means of detecting $\mathrm{dMMR}$ but the technique does not indicate which $M M R$ gene is most likely to be involved. MMR protein IHC and PCR-based MSI testing are usually considered as interchangeable methods for screening tumours for dMMR. However, if the index of suspicion for a germline $M M R$ gene defect is high, for example, in a patient under investigation by a clinical genetics department for possible Lynch syndrome, they can both be applied to the same tumour. This approach can minimise the risk of missing dMMR - this could otherwise occur for example, due to the presence of a mutation that affects the function of an MMR protein but in which the enzyme is still demonstrable using IHC, or the presence of a defect in a different, rarely implicated $M M R$ gene, for example.

Next generation sequencing is becoming more commonly used as an alternative method for detecting MSI. This is often as part of a broader panel for identifying somatic mutations and can be performed on formalin-fixed and paraffin-embedded tissue-derived DNA. This method may be better suited to large scale analyses and does not require the interrogation of matched normal tissue alongside the tumour sample. ${ }^{12}$

Formal genetic mutation analysis of germline DNA is the gold standard for identifying $M M R$ gene mutations and if found, represent a powerful tool for screening family members. However, sometimes rare or novel mutations are found and these can require correlation with IHC and MSI testing in order to assess whether they are likely to be pathogenic (ie, diseasecausing) in nature.

A germline $M M R$ gene mutation confirming the diagnosis of Lynch syndrome is found in almost all patients with an appropriate family history and tumour characteristics suggesting the presence of such a mutation. Constitutional MLH-1 promoter hypermethylation may account for up to 3\% of cases of Lynch syndrome, especially in patients where there is no clear family history. ${ }^{13}$ The presence of dMMR when no germline $M M R$ gene defect is found despite the presence of supporting clinical and tumour features has been termed 'Lynch-like syndrome'. ${ }^{14}$ The mechanisms underlying dMMR in this situation are varied and could include the presence of (1) an undetectable $M M R$ gene mutation, (2) a mutation in non- $M M R$ gene that can cause MSI or (3) a somatic bi-allelic $M M R$ gene mutation or hypermethylation. ${ }^{15}$

\section{EPCAM MUTATIONS AND DMMR}

In some patients with Lynch syndrome, IHC demonstrates loss of MSH-2 expression within their tumours but a germline mutation in $\mathrm{MSH}-2$ is not identified. Instead, a germline mutation is present at the 3' end of the EPCAM gene (the gene encoding epithelial cell adhesion molecule - EpCAM), which results in hypermethylation of the $\mathrm{MSH}-2$ promoter sequence and inactivation of $\mathrm{MSH}-2 .{ }^{16}$ This mechanism accounts for 20\%-25\% of patients whose CRC show loss of MSH-2 expression but in whom no germline $\mathrm{MSH}-2$ mutation is detected-or $2 \%-3 \%$ of all Lynch syndrome cases. ${ }^{17} \mathrm{Bi}$-allelic EPCAM mutations are associated with loss of EPCAM expression on IHC in Lynch syndrome-associated tumours. ${ }^{18}$

\section{INVESTIGATION OF LOSS OF MLH-1 EXPRESSION IN CLINICAL PRACTICE}

Loss of MLH-1 expression-usually accompanied by loss of PMS-2 expression-within tumours is the most commonly encountered abnormality on MMR protein IHC. This can indicate the presence of a germline $\mathrm{MLH}-1$ mutation but much more commonly occurs due to somatic $M L H-1$ inactivation associated with hypermethylation of its promoter sequence. In CRC, DNA from tumours showing MLH-1 loss is subjected to BRAF mutation analysis. If a $B R A F$ mutation is found, the tumour is most likely to be sporadic in nature and has probably developed along the serrated pathway. If no $B R A F$ mutation is found, tumour DNA then undergoes $M L H-1$ promoter hypermethylation analysis. The presence of hypermethylation almost always indicates that the tumour is sporadic. The exception to this is constitutional hypermethylation that is, hypermethylation of germline DNA, representing a very rare cause of Lynch syndrome. In contrast, endometrial cancers lacking MLH-1 expression rarely show BRAF mutations and therefore if MLH-1 loss is found in these tumours, direct progression to $M L H-1$ promoter hypermethylation analysis is required. In the context of absent MLH-1 and PMS-2 expression and in CRC if no BRAF mutation is present, patients with CRC or endometrial cancer in which no $\mathrm{MLH}-1$ promoter hypermethylation is found should be referred for genetic testing. This is because in these settings, they are likely to possess a germline $\mathrm{MLH}-1$ mutation.

\section{MMR PROTEIN IHC IN BIOPSIES VERSUS RESECTIONS IN CRC}

In CRC, histopathologists are usually encouraged to perform MMR protein IHC on biopsy material, if possible. This can provide an MMR status earlier in the patient pathway and rapid formalin fixation of small biopsy tissue fragments results in optimised antigen preservation and therefore minimised technical artefacts on IHC. However, sometimes IHC on biopsies is not possible for example, if the biopsy shows features that are not diagnostic of adenocarcinoma, or if an emergency colorectal resection is performed without prior biopsy for example, due to bowel perforation or obstruction. In these situations, testing must instead be undertaken on resection material.

Caution must be applied when interpreting MMR protein IHC in resection specimens from patients who have undergone neoadjuvant therapy. The latter can lead to loss of MMR protein expression in the absence of an associated germline MMR mutation and is seen most commonly with MSH-6. Therefore, if loss of expression is found when examining a post-neoadjuvant therapy specimen, repeat MMR IHC on pretreatment biopsy material is required if this is available. ${ }^{19}$

\section{MMR PROTEIN IHC IN COLORECTAL ADENOMAS VERSUS CRC}

In colorectal neoplasia, while IHC and MSI testing for dMMR is usually undertaken on biopsy or resection material showing adenocarcinoma, useful information may sometimes be gained in patients under investigation for possible Lynch syndrome, via IHC performed on colorectal adenomas. This testing is sometimes requested by clinical genetics departments, for individuals from possible Lynch syndrome families who have had colorectal adenomas removed but who do not have CRC. In patients with known Lynch syndrome, colorectal adenomas show loss of MMR protein expression in around $80 \%$ of cases. ${ }^{20}$ Therefore IHC performed on adenomas can be informative if loss of MMR protein expression is demonstrated, but this test does not exclude the possibility of Lynch syndrome if expression is retained.

Loss of MMR protein expression can be seen in morphologically normal colonic mucosa in patients with Lynch syndrome but is only very exceptionally seen in individuals without this condition. This phenomenon can provide useful supporting evidence for the presence of Lynch syndrome, particularly in patients where germline $M M R$ gene mutation analysis is inconclusive. ${ }^{21} 22$ 


\section{NATIONAL INSTITUTE FOR HEALTH AND CARE EXCELLENCE GUIDELINES FOR LYNCH SYNDROME SCREENING}

The National Institute for Health and Care Excellence (NICE) published guidelines in 2017, indicating that all patients newly diagnosed with CRC should undergo screening for Lynch syndrome, with either MMR protein IHC or MSI testing as the initial investigative method. ${ }^{23}$ Until this time, MMR protein IHC was usually performed on CRC if the clinical features (eg, patient age $<50$ years) and/or the histological features of the tumour (eg, right-sided, poorly differentiated, mucinous or tumourinfiltrating lymphocyte-rich) raised the possibility of Lynch syndrome. Additional funding was not provided for pathology laboratories to support this development. Individual patient consent is not required for this screening process, unless and until direct testing for a germline mutation is needed. Implementation of these guidelines also requires a well-defined pathway for the further investigation of MLH-1-deficient CRC and a reliable mechanism for ensuring that patients in whom screening suggests the presence of Lynch syndrome are appropriately referred to clinical genetics departments. In 2020, NICE introduced similar guidelines for patients with endometrial cancer. ${ }^{24}$

\section{DMMR AND ONCOLOGICAL CANCER TREATMENT}

In CRC, the presence of dMMR is associated with a better stageadjusted prognosis compared with MMR-proficient tumours, but a reduced likelihood of response to certain conventional chemotherapy regimens, for example, 5 -fluorouracil $\left(5\right.$-FU). ${ }^{25}$ The reason for this lack of benefit from 5-FU therapy is unknown. However, patients with CRC showing dMMR (especially stage 2, ie, pT3N0 or pT4N0) should not receive adjuvant therapy with this agent. In contrast, patients with endometrial cancer showing dMMR may show an improved response to adjuvant radiotherapy. ${ }^{26}$

The introduction of immunotherapy has improved clinical outlook within a range of cancers and the number of cancer types in which immunotherapy has been shown to be of benefit is ever-increasing. These treatments are based on boosting an antitumour immune response by patients' own immune systems, usually by blocking molecular mechanisms that tumours use to evade host attack. The presence of dMMR leads to an increased mutational burden and the generation of novel peptide sequences by cancer cells, representing an enhanced range of epitopes that are potentially recognisable by the host immune system. Therefore, tumours with $\mathrm{dMMR}$ may respond more favourably to immunotherapy than those lacking this feature.

Immunotherapies target cell surface molecules such as CTLA-4 and the PD-1/PD-L1 system. ${ }^{27}$ Of these, ipilimumab therapy for malignant melanoma, pembrolizumab therapy for non-small cell lung cancer and atezolizumab therapy for bladder cancer are already established treatments. More recent studies have demonstrated benefits of immunotherapy in a range of other advanced cancers with $\mathrm{dMMR}$, for example, pembrolizumab in CRC (KEYNOTE-177 phase III trial) and in a range of nonCRCs including endometrial, gastric, cholangiocarcinoma and pancreatic (KEYNOTE-158 phase II trial). ${ }^{28} 29$

\section{CONCLUSION}

MMR proteins play a critical role in DNA repair and therefore help protect against the accrual of sporadic mutations and the development of neoplasia. Lynch syndrome was originally described over 50 years ago but the factors leading to dMMR and its association with Lynch syndrome and 'Lynch-like' syndromes have been the subject of ongoing and intense study.
Nowadays, a detailed understanding of the biology of dMMR is essential not only for the identification of inherited predisposition to cancer but also for guiding oncologists with treatment choices associated with both conventional chemotherapy and novel immunotherapies.

Handling editor Des Richardson.

Contributors I am the sole author of this script.

Funding The author has not declared a specific grant for this research from any funding agency in the public, commercial or not-for-profit sectors.

Competing interests None declared.

Patient consent for publication Not required.

Provenance and peer review Not commissioned; externally peer reviewed.

ORCID iD

Adrian C Bateman http://orcid.org/0000-0003-2222-4104

\section{REFERENCES}

1 Pal T, Permuth-Wey J, Sellers TA. A review of the clinical relevance of mismatch-repair deficiency in ovarian cancer. Cancer 2008;113:733-42.

2 Tamura K, Kaneda M, Futagawa M, et al. Genetic and genomic basis of the mismatch repair system involved in Lynch syndrome. Int J Clin Oncol 2019;24:999-1011.

3 Lynch HT, Shaw MW, Magnuson CW, et al. Hereditary factors in cancer. Study of two large midwestern kindreds. Arch Intern Med 1966;117:206-12.

4 Latham A, Srinivasan P, Kemel Y, et al. Microsatellite instability is associated with the presence of Lynch syndrome pan-cancer. J Clin Oncol 2019;37:286-95.

5 Porkka NK, Olkinuora A, Kuopio T, et al. Does breast carcinoma belong to the Lynch syndrome tumor spectrum? - Somatic mutational profiles vs. ovarian and colorectal carcinomas. Oncotarget 2020;11:1244-56.

6 Ramchander NC, Ryan NAJ, Crosbie EJ, et al. Homozygous germ-line mutation of the PMS2 mismatch repair gene: a unique case report of constitutional mismatch repair deficiency (CMMRD). BMC Med Genet 2017;18:40.

7 Pinto D, Pinto C, Guerra J, et al. Contribution of MLH1 constitutional methylation for Lynch syndrome diagnosis in patients with tumor MLH1 downregulation. Cancer Med 2018;7:433-44.

8 Shia J, Tang LH, Vakiani E, et al. Immunohistochemistry as first-line screening for detecting colorectal cancer patients at risk for hereditary nonpolyposis colorectal cancer syndrome: a 2-antibody panel may be as predictive as a 4-antibody panel. Am J Surg Pathol 2009;33:1639-45.

9 Hall G, Clarkson A, Shi A, et al. Immunohistochemistry for PMS2 and MSH6 alone can replace a four antibody panel for mismatch repair deficiency screening in colorectal adenocarcinoma. Pathology 2010;42:409-13.

10 Mojtahed A, Schrijver I, Ford JM, et al. A two-antibody mismatch repair protein immunohistochemistry screening approach for colorectal carcinomas, skin sebaceous tumors, and gynecologic tract carcinomas. Mod Pathol 2011;24:1004-14.

11 Joost $\mathrm{P}$, Veurink N, Holck S, et al. Heterogenous mismatch-repair status in colorectal cancer. Diagn Pathol 2014;9:126.

12 Pabla S, Andreas J, Lenzo FL, et al. Development and analytical validation of a next-generation sequencing based microsatellite instability (MSI) assay. Oncotarget 2019;10:5181-93.

13 Ponti G, Manfredini M, Tomasi A, et al. Muir-Torre syndrome and founder mismatch repair gene mutations: a long gone historical genetic challenge. Gene 2016;589:127-32.

14 Carethers JM, Stoffel EM. Lynch syndrome and Lynch syndrome mimics: the growing complex landscape of hereditary colon cancer. World I Gastroenterol 2015:21:9253-61.

15 Haraldsdottir S, Hampel H, Tomsic J, et al. Colon and endometrial cancers with mismatch repair deficiency can arise from somatic, rather than germline, mutations. Gastroenterology 2014;147:1308-16

16 Ligtenberg MJL, Kuiper RP, Geurts van Kessel A, et al. EPCAM deletion carriers constitute a unique subgroup of Lynch syndrome patients. Fam Cancer 2013;12:169-74

17 Rumilla K, Schowalter KV, Lindor NM, et al. Frequency of deletions of EPCAM (TACSTD1) in MSH2-associated Lynch syndrome cases. J Mol Diagn 2011;13:93-9.

18 Huth C, Kloor M, Voigt AY, et al. The molecular basis of EPCAM expression loss in Lynch syndrome-associated tumors. Mod Pathol 2012;25:911-6.

19 Bao F, Panarelli NC, Rennert $\mathrm{H}$, et al. Neoadjuvant therapy induces loss of MSH6 expression in colorectal carcinoma. Am J Surg Pathol 2010;34:1798-804.

20 Sekine S, Mori T, Ogawa R, et al. Mismatch repair deficiency commonly precedes adenoma formation in Lynch syndrome-associated colorectal tumorigenesis. Mod Pathol 2017:30:1144-51.

21 Ahadova A, Gallon R, Gebert J, et al. Three molecular pathways model colorectal carcinogenesis in Lynch syndrome. Int J Cancer 2018;143:139-50. 


\section{Molecules in pathogenesis}

22 Pai RK, Dudley B, Karloski E, et al. DNA mismatch repair protein deficient nonneoplastic colonic crypts: a novel indicator of Lynch syndrome. Mod Pathol 2018;31:1608-18.

23 NICE. Molecular testing strategies for Lynch syndrome in people with colorectal cancer. Diagnostics guidance, 2017. Available: https://www.nice.org.uk/guidance/ dg27

24 NICE. Testing strategies for Lynch syndrome in people with endometrial cancer. Diagnostics guidance, 2020. Available: https://www.nice.org.uk/guidance/dg42

25 Kawakami H, Zaanan A, Sinicrope FA. MSI testing and its role in the management of colorectal cancer. Curr Treat Options Oncol 2015;16:30.

26 Reijnen C, Küsters-Vandevelde HVN, Prinsen CF, et al. Mismatch repair deficiency as a predictive marker for response to adjuvant radiotherapy in endometrial cancer. Gynecol Oncol 2019;154:124-30.
27 Bateman AC. Molecules in cancer immunotherapy: benefits and side effects. J Clin Pathol 2019;72:20-4.

28 André T, Shiu K-K, Kim TW, et al. Pembrolizumab in microsatellite-instability-high advanced colorectal cancer. N Engl J Med 2020;383:2207-18.

29 Marabelle A, Le DT, Ascierto PA, et al. Efficacy of pembrolizumab in patients with Noncolorectal high microsatellite Instability/Mismatch repair-deficient cancer: results from the phase II KEYNOTE-158 study. J Clin Oncol 2020;38:1-10.

30 Ferreira I, Wiedemeyer K, Demetter P, et al. Update on the pathology, genetics and somatic landscape of sebaceous tumours. Histopathology 2020;76:640-9.

31 Weissman SM, Bellcross C, Bittner CC, et al. Genetic counseling considerations in the evaluation of families for Lynch syndrome--a review. J Genet Couns 2011;20:5-19. 